\title{
COMBINED TWO STABILITIES IMPLY AXIOM A FOR VECTOR FIELDS
}

\author{
LAN WEN
}

It is proved in this paper that if a vector field is both $C^{1}$ structurally stable and topologically stable, then it satisfies Axiom A.

\section{INTRODUCTION}

Let $M$ be a compact Riemannian manifold without boundary. In this note we present a proof of the following Theorem A. See Section 2 for precise definitions.

THEOREM A. If a vector field $X$ of $M$ is both $C^{1}$ structurally stable and topologically stable, then $X$ satisfies Axiom $A$.

Note that by combining Theorem $A$ with earlier results of Nitecki $[10]$ and Robinson $[13,14,15]$, it follows immediately that $X$ is $C^{1}$ structurally stable and topologically stable if and only if $X$ satisfies Axiom A and the strong transversality condition. See [5] for more details about this.

For the case of diffeomorphisms, Theorem A was established by Hurley [5], and considerably strengthened later by Mañe [9] who solved the famous $C^{1}$ stability conjecture of Palis and Smale [12] which reduced to proving that $C^{1}$ structural stability alone implies Axiom A. We note that for the case of vector fields, the $C^{1}$ stability conjecture still remains open if $\operatorname{dim} M \geqslant 4$, and what we do in the present note is to generalise Hurley's result to the case of vector fields. We point out that we are not the one who first tried to do this. In fact, Chi, Choi and Park[1] have recently given a proof for Theorem A. Unfortunately, the proof given in [1] contains some serious gaps as we point out below, and the aim of the present note is to provide a way of making it up.

The main reason the proof in [1] falls short is that there is a delicate difference between the case of vector fields and that of diffeomorphisms regarding the results of Liao [6] and Mañé [8]. This is the type of problem of "separation implies Axiom A". To be more precise, let $\mathcal{X}^{1}(M)$ be the set of $C^{1}$ vector fields of $M$, and $\mathcal{X}^{*}(M)$ be the set of vector fields $X$ that have a $C^{1}$ neighbourhood $\mathcal{U}$ in $\mathcal{X}^{1}(M)$ such that all singularities and all periodic orbits of every $Y \in \mathcal{U}$ are hyperbolic. Let $\operatorname{Sing}(X)$

Received 16th July, 1992

The author wishes to thank Professor T. Ding for references and helpful communications.

Copyright Clearance Centre, Inc. Serial-fee code: 0004-9729/93 \$A2.00+0.00. 
be the set of singularities of $X$, and $\Lambda_{i}(X)$ be the closure of the union of hyperbolic periodic orbits of $X$ whose stable manifolds have dimension $i$. Similarly, let $\operatorname{Diff}^{1}(M)$ be the set of $C^{1}$ diffeomorphisms of $M$, and $\mathcal{F}^{*}(M)$ be the set of diffeomorphisms $f$ that have a $C^{1}$ neighbourhood $\mathcal{U}$ in $\operatorname{Diff}^{1}(M)$ such that all periodic points of every $g \in \mathcal{U}$ are hyperbolic. Let $\Lambda_{i}(f)$ be the closure of the set of hyperbolic periodic points of $f$ whose stable manifolds have dimension $i$. An important theorem obtained by Liao [6] was the following

Theorem L. (Liao). Assume that there is a $C^{1}$ neighbourhood $\mathcal{W}$ of $X$ in $\mathcal{X}^{*}(M)$ together with some mutually disjoint open sets $G$ and $W_{i}$ of $M, i=$ $1, \cdots, \operatorname{dim} M$, such that for every $Y \in \mathcal{W}$ one has $\operatorname{Sing}(Y) \subset G$ and $\Lambda_{i}(Y) \subset$ $W_{i}, i=1, \cdots, \operatorname{dim} M$. Then $X$ satisfies Axiom $A$.

Not long after, Mañé [8] obtained another important theorem of this type for the case of diffeomorphisms:

Theorem M. (Mañé). Assume that $f$ is in $\mathcal{F}^{*}(M)$ and $\Lambda_{i}(f) \cap \Lambda_{j}(f)=\phi$ for all $0 \leqslant i<j \leqslant \operatorname{dim} M$. Then $f$ satisfies Axiom $A$.

Note that the separation assumption made in Theorem $M$ for diffeomorphisms is weaker than that in Theorem $L$ for vector fields. Hence it remained for some time an important question whether or not Theorem $\mathrm{L}$ could be strengthened to the following statement which is an analogue of Theorem $M$ for the case of vector fields.

Statement S: If $X$ is in $\mathcal{X}^{*}(M)$, and if $\operatorname{Sing}(X)$ and $\Lambda_{i}(X), i=1, \cdots, \operatorname{dim} M$, are mutually disjoint, then $X$ satisfies Axiom $A$.

Strikingly enough, statement $\mathrm{S}$ was shown false by an example of Ding [2]. This was a warning that there are some delicate differences between $\mathcal{X}^{*}$ and $\mathcal{F}^{*}$. See [7] for more comments about this.

Unfortunately, it is statement $S$ that is assumed as a major argument to prove Theorem A. Thus the proof is incomplete. (This does not affect the proof of Hurley [5] because he used Theorem M.) In the present note we shall use Theorem $\mathrm{L}$ to fill the gap. Another point which is missed in [1] is the problem of dealing with singularities. This will be made up by Lemma 4 of Section 3 below.

\section{Preliminaries}

Let $\mathcal{X}^{1}(M)$ be the set of $C^{1}$ vector fields on $M$. We say that $Y \in \mathcal{X}^{1}(M)$ is semiconjugate to $X \in \mathcal{X}^{1}(M)$ if there is a continuous surjection $h: M \rightarrow M$ and a continuous map $r: M \times \mathbb{R} \rightarrow \mathbb{R}$ such that:

(1) for all $x \in M, \tau(x, 0)=x$, and $\tau(x, \bullet): \mathbb{R} \rightarrow \mathbb{R}$ is an orientation preserving homeomorphism; 
(2) for all $x \in M$ and $t \in \mathbb{R}, h(\psi(x, t))=\phi(h x, \tau(x, t))$, where $\phi$ and $\psi$ are the flows induced by $X$ and $Y$ respectively.

The pair $(h, \tau)$, or more briefly, the surjection $h$, is called a semiconjugacy from $Y$ to $X$. If $h$ can be taken as a homeomorphism, we say that $Y$ is conjugate, or topologically equivalent, to $X$, and $h$ is a conjugacy, or a topological equivalence.

We shall consider both the $C^{\circ}$ and the $C^{1}$ topology on $\mathcal{X}^{1}(M) . X$ is called $C^{1}$ structurally stable if there is a $C^{1}$ neighbourhood $\mathcal{U}$ of $X$ in $\mathcal{X}^{1}(M)$ such that any $Y \in \mathcal{U}$ is conjugate to $X . X$ is called topologically stable if for any $\varepsilon>0$, there is a $C^{\circ}$ neighbourhood $\mathcal{U}$ of $X$ in $\mathcal{X}^{1}(M)$ such that for any $Y \in \mathcal{U}$, there is a semiconjugacy $h=h_{Y}$ from $Y$ to $X$ with $d(h x, x)<\varepsilon$ for any $x \in M$, where $d$ is the distance induced by the Riemannian metric. Note that in the definition of topological stability an arbitrarily small $\varepsilon$ is involved. This will play a crucial role in the final proof of Theorem A below.

Let $X \in \mathcal{X}^{1}(M)$ and $\phi_{t}=\phi(\bullet, t)$ be the induced flow of $X$. A point $x \in M$ is a non-wandering point of $X$ if for any neighbourhood $U$ of $x$ in $M$, there is a $t \geqslant 1$ such that $\phi_{t}(U) \cap U \neq \phi$. The set of non-wandering points of $X$ is denoted $\Omega(X)$. Clearly, the singularities and the points of periodic orbits are all non-wandering. We say that $X$ satisfies Axiom $A$ if the following two conditions are satisfied.

Axiom A(a). The tangent flow $d \phi_{t}: T M \rightarrow T M$ leaves invariant a continuous splitting $T_{\Omega(X)} M=E^{*} \oplus \operatorname{Span} X \oplus E^{u}$ such that, for some constants $C>0$ and $\lambda>0$,

$$
\begin{aligned}
& \left\|d \phi_{t} \mid E^{\bullet}(x)\right\| \leqslant C \exp (-\lambda t), \\
& \left\|d \phi_{t} \mid E^{u}(x)\right\| \leqslant C \exp (-\lambda t)
\end{aligned}
$$

for any $t>0$ and $x \in \Omega(X)$.

Axiom $\mathrm{A}(\mathrm{b})$. The periodic orbits are dense in $\Omega(X)-\operatorname{Sing}(X)$.

Note that if $X$ satisfies Axiom $A$, then the singularities are bounded away from the periodic orbits by the continuity of the invariant splitting.

Denote

and

$$
W^{\prime}(x)=\left\{y \in M \mid d\left(\phi_{t}(y), \phi_{t}(x)\right) \rightarrow 0 \text { as } t \rightarrow \infty\right\}
$$

$$
W^{u}(x)=\left\{y \in M \mid d\left(\phi_{t}(y), \phi_{t}(x)\right) \rightarrow 0 \text { as } t \rightarrow-\infty\right\} \text {. }
$$

We call $W^{\prime}(x)$ and $W^{u}(x)$ the stable and the unstable manifold of $x$, respectively. If $A$ is a subset of $M$, define $W^{\bullet}(A)$ to be the union of $W^{\bullet}(x)$ for $x \in A$. Similarly we define $W^{u}(A)$. If $\gamma$ is a hyperbolic periodic orbit of $X$, then $W^{\bullet}(\gamma)$ and $W^{u}(\gamma)$ are immersed submanifolds of $M$ with $\operatorname{dim} W^{\iota}(\gamma)+\operatorname{dim} W^{u}(\gamma)=\operatorname{dim} M+1$. We call $\operatorname{dim} W^{\prime}(\gamma)$ the index of $\gamma$. If $X$ satisfies Axiom $\mathrm{A}$, then for any $x \in M, W^{\prime}(x)$ and 
$W^{u}(x)$ are immersed submanifolds of $M$. In this case we say that $X$ satisfies the strong transversality condition if $W^{u}(x)$ is transverse to $W^{u}(x)$ at any $x \in M$. It is well known that if $X$ satisfies Axiom $A$ and the strong transversality condition, then $X$ is both $C^{1}$ structurally stable and topologically stable $[10,14,15]$.

Let $x, y \in M$ and $\varepsilon, T>0$ be given. A finite sequence $\left\{\left(x_{i}, t_{i}\right)\right\}, i=1, \cdots, n$, in $M \times \mathbb{R}$ is called an $(\varepsilon, T)$-chain from $x$ to $y$ if $x_{1}=x, t_{i} \geqslant T$ for $i=$ $1, \cdots, n, d\left(\phi\left(x_{i}, t_{i}\right), x_{i+1}\right)<\varepsilon$ for $i=1, \cdots, n-1$, and $d\left(\phi\left(x_{n}, t_{n}\right), y\right)<\varepsilon$. Denote $x \sim y$ if for any $\varepsilon, T>0$, there is an $(\varepsilon, T)$-chain from $x$ to $y$ and an $(\varepsilon, T)$-chain from $y$ to $x$. A point $x$ is chain recurrent if $x \sim x$. Let CR(X) denote the set of chain recurrent points of $X$. Clearly, $\sim$ is an equivalence relation on $C R(X)$. The equivalence classes are called the chain components of $X$. The chain components of $X$ are disjoint, closed, invariant sets.

\section{The proof of Theorem A}

In this section we prove Theorem A. The route of the proof will be essentially that of Hurley [5] with some specific consideration put on the singularities.

By virtue of Theorem $L$ quoted in Section 1, the proof of Theorem $A$ reduces to proving that if $X$ is both $C^{1}$ structurally stable and topologically stable, then $X$ satisfies the assumptions of Theorem $L$. To this end we shall need the following four lemmas. Three of them have been essentially obtained by Hurley [4, 5], and the fourth one is an easy application of the $C^{\circ}$ perturbation technique.

Lemma 1. ([4]) Assume that $X$ is topologically stable. Then the union of the singularities and periodic orbits of $X$ is dense in the chain recurrent set of $X$. Moreover, $X$ has only finitely many chain components.

LEMma 2. Assume that $X$ is topologically stable, and that $Y$ is semiconjugate to $X$ by a semiconjugacy $h$. Then $h$ maps each chain component of $Y$ into some chain component of $X$, and each chain component of $X$ is the $h$-image of some chain component of $Y$.

For the case of diffeomorphisms, Lemma 2 was proved in [5]. The proof works for vector fields equally well.

LEMMA 3. Let $X$ be both $C^{1}$ structurally stable and topologically stable, and $C$ be any chain component of $X$ which is not just a single point. Then all periodic orbits of $C$ have the same index.

The proof of Lemma 3 given below is a slight modification of the proof given in [5] for the case of diffeomorphisms. A refined version of Lemma 3 is proved in [1].

Proof: Since $X$ is topologically stable, there is a $C^{\circ}$ neighbourhood $\mathcal{U}$ of $X$ in $\mathcal{X}^{1}(M)$ such that each $Y \in \mathcal{U}$ is semiconjugate to $X$ by a continuous surjection 
$h$. By Shub's $C^{\circ}$ density theorem [16] we can find a $Y \in \mathcal{U}$ such that $Y$ satisfies Axiom $\mathrm{A}$ and the strong transversality condition. It is standard that in this case $\mathrm{CR}(Y)=\Omega(Y)$, and the chain components of $Y$ are exactly those basic sets of $Y$ in the spectural decomposition of $\Omega(Y)$. Let $C$ be any chain component of $X$ which is not just a single point. By Lemma 2, there is a basic set, say $\Omega_{1}$, of $Y$ such that $h\left(\Omega_{1}\right)=C$. It is a standard fact about basic sets that, for any two points $x$ and $y$ in $\Omega_{1}$, whether on periodic orbits or not, one has $W^{u}(O(x)) \cap W^{u}(O(y)) \neq \phi$, where $O(x)$ and $O(y)$ denote the $Y$-orbit of $x$ and $y$ respectively. It follows from the semiconjugacy that for any two periodic orbits $\gamma_{1}$ and $\gamma_{2}$ of $C, W^{\prime}\left(\gamma_{1}\right) \cap W^{u}\left(\gamma_{2}\right) \neq \phi$. Since $X$ is $C^{1}$ structurally stable, the intersections of $W^{\prime}\left(\gamma_{1}\right)$ and $W^{u}\left(\gamma_{2}\right)$ are all tranverse [13]. Then $\gamma_{1}$ and $\gamma_{2}$ must have the same index. In fact, if we denote $s_{i}=$ $\operatorname{dim} W^{\boldsymbol{s}}\left(\gamma_{i}\right), u_{i}=\operatorname{dim} W^{u}\left(\gamma_{i}\right), i=1,2$, then $s_{1}+u_{1}=s_{2}+u_{2}=\operatorname{dim} M+1$. Moreover, $s_{1}+u_{2} \geqslant \operatorname{dim} M+1, s_{2}+u_{1} \geqslant \operatorname{dim} M+1$ by transversality. Thus $s_{1}=s_{2}, u_{1}=u_{2}$.

As Hurley [5] observed, a direct consequence of Lemma 3 is that, if $X$ is both $C^{\mathbf{1}}$ structurally stable and topologically stable, then $\Lambda_{i}(X) \cap \Lambda_{j}(X)=\phi$ for $1 \leqslant i<$ $j \leqslant \operatorname{dim} M$. Note that it is still not yet clear in this case if the periodic orbits could accumulate on the singularities. The following lemma deals with this, which is missed in $[\mathbf{1}]$.

Lemma 4. If $X$ is both $C^{1}$ structrually stable and topologically stable, then $\operatorname{Sing}(X) \cap \Lambda_{i}(X)=\phi$ for all $i=1, \cdots, \operatorname{dim} M$.

Proof: If $\operatorname{dim} M=2$, Lemma 4 is certainly true according to Peixoto's classical results [11]. Hence we shall assume $\operatorname{dim} M \geqslant 3$ below.

Suppose for contradiction that there is a sequence of periodic orbits $\gamma_{1}, \gamma_{2}, \ldots$ of $X$ that accumulate on a singularity $\sigma$ of $X$, that is, there is a sequence of points $z_{i_{k}} \in \gamma_{i_{k}}$ such that $z_{i_{k}} \rightarrow \sigma$. Clearly, $\sigma$ must be a saddle. We shall find a $C^{1}$ vector field $Y$, arbitrarily $C^{\circ}$ close to $X$ and coinciding with $X$ in a neighbourhood of $\sigma$, such that $Y$ has a homoclinic orbit associated with $\gamma$. Then since $X$ is topologically stable, $Y$ is semiconjugate to $X$ by some semiconjugacy, which would give back a homoclinic orbit of $X$ associated with a singularity of $X$. But $X$ is also $C^{1}$ structurally stable and hence can not have any homoclinic orbits. This contradiction will complete the proof of Lemma 4. Thus it remains to find such a $C^{\circ}$ perturbation $Y$. This is just an easy application of the $C^{\circ}$ perturbation technique. We only sketch the way the perturbation is made.

Let $S^{\boldsymbol{s}}$ and $S^{u}$ be two small closed balls of center $\sigma$ in $W^{\boldsymbol{s}}(\sigma)$ and $W^{u}(\sigma)$, respectively. By compactness of $S^{\boldsymbol{u}}$ and $S^{u}$ there are two points $p \in S^{u}$ and $q \in S^{*}$ such that $\left\{\gamma_{i}\right\}$ accumulates on both $p$ and $q$. About $p$ and $q$ we take two small flow boxes $B_{p}=\Sigma_{p} \times I$ and $B_{q}=\Sigma_{q} \times J$, where $\Sigma_{p}$ and $\Sigma_{q}$ are local transversals. We can find a subarc $[x, y]$ of some $\gamma_{i}$, leaving $B_{p}$ at $x$ and entering $B_{q}$ at $y$, see figure 1 . 
There are probably some intermediate intersections of $[x, y]$ with the two flow boxes.

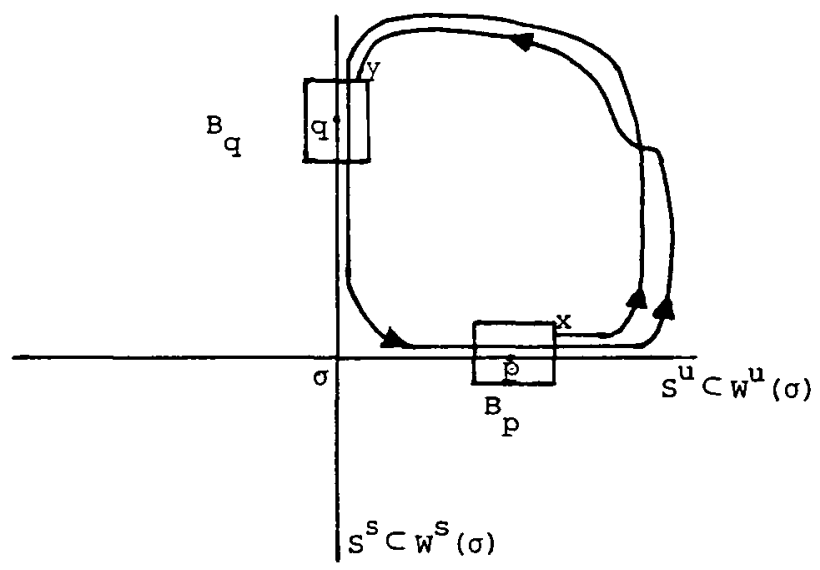

Now a standard way of $C^{\circ}$ perturbation creates a $C^{1}$ vector field $Y$, arbitrarily $C^{\circ}$ close to $X$ and coinciding with $X$ outside $B_{p}$ and $B_{q}$, such that $Y$ has a homoclinic orbit associated with $\sigma$. The intermediate intersections will not affect the perturbation process since we have assumed $\operatorname{dim} M \geqslant 3$ and hence $\operatorname{dim} \Sigma_{p}=\operatorname{dim} \Sigma_{q} \geqslant 2$. In fact, the perturbation can be made so that the positive $Y$-orbit of $p$ hits $x$ before leaving $B_{p}$, then goes through $[x, y]$ to get to $y$ since $[x, y]$ remains unperturbed, and finally hits $q$ right afterwards.

Now we prove the main result of this paper.

TheOREM A. If $X$ is both $C^{1}$ structurally stable and topologically stable, then $X$ satisfies Axiom $A$.

Proof: By Lemma 1, $X$ has only finitely many chain components $C_{1}, \cdots, C_{p}$, each being the closure of a union of some singularities and periodic orbits. By Lemma 4, singularities are bounded away from periodic orbits. Thus each singularity itself forms a chain component of $X$, and each of the other chain components has, by Lemma 3 , a unique value of the index of periodic orbits. Without loss of generality we may assume that the subscripts of $C_{j}$ have been arranged so that $C_{1}, \cdots, C_{k_{0}}$ are each a singularity, and $C_{k_{0}+1}, \cdots, C_{k_{1}}$ are the chain components whose periodic orbits have index 1 , and so on. In other words, $\operatorname{Sing}(X)=\left\{C_{1}, \cdots, C_{k_{0}}\right\}$, and $\Lambda_{i}(X)$ is the union of $C_{j}, j=k_{i-1}+1, \cdots, k_{i}$. In particular, $\operatorname{Sing}(X)$ and $\Lambda_{i}(X)$ are mutually disjoint, $i=1, \cdots, \operatorname{dim} M$.

Let $\varepsilon>0$ be small enough such that the $\varepsilon$-neighbourhoods of these sets, $N_{\varepsilon}(\operatorname{Sing}(X)), N_{\varepsilon}\left(\Lambda_{i}(X)\right), i=1, \cdots, \operatorname{dim} M$, are mutually disjoint. For this $\varepsilon$, there 
is a $C^{1}$ neighbourhood $\mathcal{W}$ of $X$ in $\mathcal{X}^{*}(M)$ such that

(1) each $Y \in \mathcal{W}$ is conjugate (that is, topologically equivalent) to $X$, and is semiconjugate to $X$ by a semiconjugacy $h$ with $d(h, i d)<\varepsilon / 2$;

(2) each $Y \in \mathcal{W}$ is both $C^{1}$ structurally stable and topologically stable.

The existence of such a $\mathcal{W}$ follows directly from the definitions of $C^{1}$ structural stability and topological stability, together with the two standard facts that any $C^{1}$ structurally stable system is in $\mathcal{X}^{*}$ (see [3]), and that topological stability is a conjugacy invariant. There will be in fact an additional condition we want $\mathcal{W}$ to satisfy, see the end of the proof. Now denote

$$
G=N_{\varepsilon / 2}(\operatorname{Sing}(X))
$$

and

$$
W_{i}=N_{\varepsilon / 2}\left(\Lambda_{i}(X)\right), \quad i=1, \cdots, \operatorname{dim} M .
$$

We shall prove that for any $Y \in \mathcal{W}$, one has $\operatorname{Sing}(Y) \subset G$, and $\Lambda_{i}(Y) \subset W_{i}, i=$ $1, \cdots, \operatorname{dim} M$. By Theorem $\mathrm{L}$ quoted in Section 1 , this will complete the proof of theorem $A$. Let $Y \in \mathcal{W}$ by given, and let $h$ be the semiconjugacy from $Y$ to $X$.

Since $Y$ is conjugate to $X, X$ and $Y$ have the same number of chain components, and the same number of singularities. Let $D_{1}, \cdots, D_{p}$ be the chain components of $Y$, and $D_{1}, \cdots, D_{k_{0}}$ be the sigularities of $Y$. By Lemma $2, h$ induces a bijection map from $\left\{D_{1}, \cdots, D_{p}\right\}$ to $\left\{C_{1}, \cdots, C_{p}\right\}$, because $p$ is a finite number. Note that $h$ must map singularities to singularites. Rearranging the subscripts of $D_{j}$ if necessary, we may assume that $h\left(D_{j}\right)=C_{j}$ for $j=1, \cdots, p$. Since $d(h, i d)<\varepsilon / 2$ it follows that $D_{j} \subset$ $N_{\varepsilon / 2}\left(C_{j}\right)$. Thus $\operatorname{Sing}(Y) \subset G$. To see that $\Lambda_{i}(Y) \subset W_{i}$ for $i=1, \cdots, \operatorname{dim} M$, it suffices to prove that, for each $j=k_{0}+1, \cdots, p$, the periodic orbits of $D_{j}$ and $C_{j}$ have the same index. And by Lemma 3, this reduces to proving that for each $j=k_{0}+1, \cdots, p$, there is a periodic orbit $\gamma_{j}$ of $C_{j}$ and a periodic orbit $\beta_{j}$ of $D_{j}$ such that $\gamma_{j}$ and $\beta_{j}$ have the same index. To this end we use Hartman's theorem. For each $j=k_{0}+1, \cdots, p$, choose and fix a periodic orbit $\gamma_{j}$ in $C_{j}$. By Hartman's theorem, if $\mathcal{W}$ is small enough, there is a periodic orbit $\beta_{j}$ of $Y$ in the $(\varepsilon / 2)$ - neighbourhood of $\gamma_{j}$ in $M$ such that $\beta_{j}$ and $\gamma_{j}$ have the same index. Clearly $\beta_{j}$ is contained in $D_{j}$. But as remarked earlier, we could have chosen $\mathcal{W}$ this small. This completes the proof of Theorem A.

\section{REFERENCES}

[1] D.P. Chi, S.K. Choi and J.S. Park, 'Structurally stable flows', Bull. Austral. Math. Soc. 45 (1992), 79-90.

[2] H. Ding, 'Disturbance of the homoclinic trajectory and applications', (in Chinese), Acta. Sci. Natur. Univ. Pekinensis 1 (1986), 53-63. 
[3] J. Franks, 'Necessary conditions for stability of diffeomorphisms', Trans. Amer. Math. Soc. 158 (1971), 301-308.

[4] M. Hurley, 'Consequences of topological stability', J. Differential Equations 54 (1984), 60-72.

[5] M. Hurley, 'Combined structural and topological stability are equivalent to Axiom $A$ and the strong transversality condition', Ergodic Theory Dynamical Systems 4 (1984), 81-88.

[6] S. Liao, 'Obstruction sets (II)', (in Chinese), Acta Sci. Natur. Univ. Pekinensis 2 (1981), 1-36.

[7] S. Liao, 'Obstruction sets, minimal rambling sets and their applications', in Chinese Mathematics into the $21^{\text {st }}$ Century (Peking Univ. Press, 1991), pp. 1-14.

[8] R. Mañé, 'An ergodic closing lemma', Ann. of Math. 116 (1982), 503-540.

[9] R. Máne, 'A proof of the $C^{1}$ stability conjecture', Publ. Math. I.H.E.S. 66 (1987), 161-210.

[10] Z. Nitecki, 'On semistability for diffeomorphisms', Invent. Math. 14 (1971), 83-122.

[11] M.M. Peixoto, 'Structural stability on two-dimensional manifolds', Topology 1 (1962), 101-120.

[12] J. Palis and S. Smale, 'Structural stability theorems', in Global Analysis, Proc. Sympos. Pure Math. 14 (American Mathematical Society, Providence, Rhode Island, 1970), pp. 223-231.

[13] C. Robinson, ' $C$ ' structural stability implies Kupka-Smale', in Dynamical systems, Salvador 1971 (Academic Press, 1973), pp. 443-449.

[14] C. Robinson, 'Structural stability of vector fields', Ann. of Math 99 (1974), 154-175.

[15] C. Robinson, 'Stability theorems and hyperbolicity in dynamical systems', Rocky Mountain J. Math. 7 (1977), 425-437.

[16] M. Shub, 'Dynamical systems, filtrations and entropy', Bull. Amer. Math. Soc. 80 (1974), 27-41.

\footnotetext{
Department of Mathematics

Peking University

Beijing 100871

China
} 